ENGINEERING RESEARCH INSTITUTE

THE UNIVERSITY OF MICHIGAN

ANN ARBOR

LATERAL BUCKIJING OF PLANE FRAMEWORKS

E. F. Masur

A. Cukurs

Project 2480

ORDNANCE CORPS, DETROIT ORDNAINCE DISTRICT

CONTRACT NO. DA-20-018-ORD-14085

DEPARTMENT OF ARMY PROJECT NO. 5B99-01-004

ORDNANCE $R$ AND D PROJECT NO. TB2-0001

OOR PROJECT NO. 1596

May 1956 



\title{
LATERAL BUCKLING OF PLANE FRAMEWORKS ${ }^{1}$
}

\author{
By E. F. Masur ${ }^{2}$ and A. Cukurs 3
}

\section{SUMMARY}

In current design practice it is generally assumed that a truss is equally safe against buckling in and out of its plane if its members have the same slenderness ratio in both directions. In this paper it is shown that the argument may be inapplicable to the customary rigid jointed type of structure. In fact it is demonstrated that this kind of truss, designed under present code requirements, may exhibit a much smaller factor of safety against buckling normal to its plane than in its plane.

The analysis of the lateral stability proceeds from the establishment of the basic equations to the derivation of a determinantal form of stability criterion. An alternate approach, pursued further in this paper because of its reduced numerical difficulty, utilizes a generalized moment distribution technique in the analysis of the response of the truss to applied unit couples whose planes are perpendicular to that of the structure. This results in the development of a "series criterion" which represents an extension and generalization of a similar criterion first proposed by Lundquist.

\section{INTRODUCTION}

In the design of a compression member of a steel truss, it is customary, under present code requirements $[1],{ }^{4}$ to consider the allowable axial stress a function of the largest slenderness ratio alone, without regard to the condition of elastic restraint at the joints. It follows therefore that an "efficient" member should display approximately the same slenderness ratio with respect to both

\footnotetext{
$I_{\text {This }}$ work was sponsored by the Office of Ordnance Research, U.S. Army. ${ }^{2}$ Associate Professor of Engineering Mechanics, The University of Michigan, Ann Arbor, Michigan.

${ }^{3}$ Structural Engineer, Procon, Incorporated, Chicago, Illinois.

${ }^{4}$ Numbers in brackets refer to the bibliography at the end of the paper.
} 

principal directions; this, it is felt, should insure an equal factor of safety against buckling both in and out of the plane of the structure.

It is fairly obvious that this kind of reasoning cannot be applied to a large and common class of trusses without major modification. Even in the event of perfect pin connections at the joints, the argument is valid only if the centroid and shear center coincide. This is often not the case. The customary double-angle type of section, for example, buckles laterally in a mode which couples bending and twisting, and under an axial load which may be appreciably smaller than the Euler load, which is based on a bending mode alone.

If the bars are held rigidly at the joints, this discrepancy is likely to increase further. When buckling occurs in the elastic region (that is, for sufficiently slender elements) the critical compressive stress depends to a large extent on the degree of end restraint. This restraint, which is imposed on one member by the adjoining members, is generally less effective relative to lateral buckling that in relation to buckling in the plane of the structure. This is especially true of double-angle sections, whose torsional stiffness is negligible compared with their bending stiffness. It is therefore not implausible to expect trusses composed of such members to exhibit a greater tendency to buckle normal to their plane than in their own plane.

It is the purpose of this paper to investigate the conditions under which this type of instability will occur. In order to facilitate the analysis, a number of assumptions are made which, it is felt, are reasonable without reducing the generality of the approach to a significant extent. It is assumed, for example, that the members of the truss are joined rigidly at the panel points; the effect of the gussetplates is therefore disregarded. It is postulated further that the panel points are held rigidly against lateral translation by means of lateral cross bracing, which, however, does not contribute to the rotational joint stiffness. Since all members are composed of two angles, the further as- 

sumption that the shear center itself is held against lateral movement at the joints appears not unwarranted in view of the fact that the cross bracing is ordinarily connected to the outstanding legs.

The analysis in the form presented here is not confined to double-angle sections; it is restricted, however, to sections whose warping stiffness may be considered negligible in comparison with their torsional and bending stiffnesses. An extension to sections exhibiting appreciable warping resistance, such as, e.g., I-beams, may be effected at the expense of some of the numerical simplicity. On the other hand, this does not appear worthwhile since members of this type are rarely slender enough to be in danger of elastic buckling, at least so far as common structural practice is concerned.

It is finally assumed that, prior to lateral buckling, all members are subjected only to axial forces through their centroids. This implies, of course, that all external loads are applied at the joints, that all bars are initially straight, and that the effect of the joint translations within the plane of the framework may be neglected. Actually, "secondary moments" are inevitable and are likely to affect the condition of lateral instability to a significant extent. Nevertheless, this omission is not considered serious, as will be discussed in the Conclusion.

\section{ESTABLISHMENT OF BASIC EQUATIONS}

In what follows, let a representative bar between joints $i$ and $j$ buckle laterally as shown in Fig. 1. The differential equations of equilibrium governing the displacements $(u, v)$ of the shear center $S$ and the rotation $\beta$ are well-known and are given, for example, in [2], p. 132. For the case under consideration, in which the warping constant is assumed to vanish and in which, by virtue of the symmetry of all sections, buckling in the plane of the frame- 

work is uncoupled and may therefore be ignored, they read:

$$
\begin{aligned}
& \mathrm{EI}_{\mathrm{y}} \mathrm{u}^{\mathrm{iv}}+\mathrm{P} \mathrm{u}^{\prime \prime}+\mathrm{P} \mathrm{y}_{\mathrm{O}} \beta^{\prime \prime}=0 \\
& \mathrm{P} \mathrm{y}_{\mathrm{O}} \mathrm{u}^{\prime \prime}+\left(\mathrm{P} \rho^{2}-\mathrm{G} \mathrm{K}\right) \beta^{\prime \prime}=0
\end{aligned}
$$

In these equations, the axial force $\mathrm{P}$ is considered positive in compression. $E$ and $G$ are the usual material constants, while $I_{y}$ and $K$ are, respectively, the moment of inertia about the $\mathrm{y}$-axis and the torsional stiffness constants. 5 The radius of gyration about the shear center is designated by $\rho$, and $y_{O}$ is the coordinate of the shear center relative to the centroid C. Primes, as usual, indicate differentiation with respect to the independent variable $(z)$ along the axis of the bar.

Eqs. (1) and (2) can be simplified somewhat. If the dimensionless quantity $\mu$ is defined by

$$
\mu=\frac{\mathrm{y}_{0}{ }^{2}}{\mathrm{GK} / \mathrm{P}-\rho^{2}}
$$

it follows, from Eq. (2), that

$$
y_{0} \beta^{\prime \prime}=\mu u^{\prime \prime}
$$

and, upon substitution in Eq. (I), that

$$
E I_{y} u^{i v}+N u^{\prime \prime}=0
$$

in which $\mathbb{N}$ is an "equivalent" axial force given by

$$
N=P(1+\mu)
$$

5 To be on the safe side, $K$ for the section is assumed to be twice the value of the individual angle. 

The general solution of these linear homogeneous equations is readily established and involves six constants of integration. Basically, this process should now be repeated with each member. There results a large number of constants of integration, for which an equal number of linear homogeneous boundary conditions is available. The problem is to determine the smallest load for which the ensuing system of linear homogeneous equations admits a non-trivial solution; in this fashion, a typical determinantal stability criterion is established.

This represents a task of formidable numerical proportions. It can be simplified greatly, however, through a systematizing procedure which is analogous to the establishment of modified "slope-deflection" equations. To this end, the bar rotations $\beta$ and $\theta$ at the joints (representing twisting and bending rotations, respectively) are introduced as independent variables, and Eqs. (4) and (5) are solved subject to the boundary conditions.

$$
\begin{array}{rlrl}
u(0) & =0 & u(L) & =0 \\
u^{\prime}(0) & =\theta_{i j} & u^{\prime}(L) & =-\theta_{j i} \\
\beta(0) & =\beta_{i j} & \beta(L) & =-\beta_{j i}
\end{array}
$$

in which $\mathrm{L}$ represents the length of the member. The choice of the sign convention is governed by considerations of consistency for the developments below.

The functions $u(z)$ and $\beta(z)$ are thus established in terms of the four end rotations; their form is of no direct interest here and has therefore been omitted. However, it is now possible to express the twisting moment $T$ and bending moment $\mathrm{M}$ at the joints in terms of these rotations. This is accomplished by letting

$$
\begin{aligned}
T_{i j} & =\left(G K-P \rho^{2}\right) \beta^{\prime}(0)-P y_{0} u^{\prime}(0) \\
T_{j i} & =\left(G K-P \rho^{2}\right) \beta^{\prime}(L)-P y_{O} u^{\prime}(L) \\
M_{i j} & =E I_{y} u^{\prime \prime}(0) \\
M_{j i} & =E I_{y} u^{\prime \prime}(L)
\end{aligned}
$$



In view of these equations, the moment-rotation relationships take the following simple form in the case of prismatic bars:

$$
\begin{aligned}
& T_{i j}=-S_{t}\left(\beta_{i j}-C_{t} \beta_{j i}\right) \\
& M_{i j}=-S_{m}\left(\theta_{i j}-C_{m} \theta_{j i}\right)
\end{aligned}
$$

with $\mathrm{T}_{j i}$ and $\mathrm{M}_{j i}$ obtained by inverting the subscripts $(i, j)$ on the right side of Eqs. (9). It is of interest to note, and is physically plausible, that the choice of the shear center as reference point has led to an uncoupling of twisting and bending, so far as the joints are concerned. From this it is not to be inferred, however, that there is no coupling all along the bar; on the contrary, pure bending at the joints, for example, produces bending and twisting, unless the centroid and shear center coincide.

In Eqs. (9), the "stiffness factors" $S_{t}$ and $S_{m}$ and the "carry-over factors" $\mathrm{C}_{t}$ and $\mathrm{C}_{\mathrm{m}}$ are given by the following equations:

$$
\begin{aligned}
& S_{t}=G K-P \rho^{2} \\
& C_{t}=-I \\
& S_{m}=\frac{E I_{y}}{L} \phi \frac{\sin \phi-\phi \cos \phi}{2-2 \cos \phi-\phi \sin \phi} \\
& C_{m}=\frac{\phi-\sin \phi}{\sin \phi-\phi \cos \phi} \\
& \phi^{2}=\frac{N L^{2}}{E I_{y}}
\end{aligned}
$$

In these equations, the trigonometric functions are replaced by the appropriate hyperbolic functions for negative values of $\mathrm{N}$. When $\mathrm{N}$ vanishes, $\mathrm{S}_{\mathrm{m}}$ and $\mathrm{C}_{\mathrm{m}}$ assume the conventional values of $4 \mathrm{EI} / \mathrm{L}$ and $1 / 2$, respectively. They have been tabulated, with slight modifications, in [3] for both tensile and compressive axial forces. With the joint moments, both in twisting and in bending, thus established in terms of the joint rotations by means of Eqs. (9), there remain two major consid- 

erations to be observed. In the first place, the rotations $\left(\beta_{i j}, \theta_{i j}\right)$ of all the bars joined at panel point $i$ must be compatible with the rigidity of the joint; in other words, the angles between the individual members must be preserved during any rotation. Secondly, the total of all the bending and twisting moments must be in equilibrium.

To this end, it has been found that the employment of matrix notation leads to compactness of both conceptual and computational labor. Consider, for example, the typical bar connecting joints $i$ and $j$ (see Fig. 2 ), with its inclination $\alpha_{i j}$ relative to the positive $x$-axis defined as shown. All rotations and couples are assumed positive as indicated by the arrows, which are selected according to the usual right-hand-screw convention. This agrees, it may be noted, with the previous choice of boundary conditions and, in particular, with Eqs. (9). If now a moment vector $\mathrm{m}^{\prime}$ and a rotation vector $\omega^{\prime}$ are defined by 6

$$
\mathrm{m}^{\prime}=\left[\begin{array}{l}
\mathrm{T} \\
\mathrm{M}
\end{array}\right] \quad \omega^{\prime}=\left[\begin{array}{l}
\beta \\
\theta
\end{array}\right]
$$

and if, further, a "stiffness matrix" $S^{\prime}$ and a "carry-over matrix" $C^{\prime}$ are introduced by means of

$$
S^{\prime}=\left[\begin{array}{cc}
S_{t} & 0 \\
0 & S_{m}
\end{array}\right] \quad C^{\prime}=\left[\begin{array}{cc}
C_{t} & 0 \\
0 & C_{m}
\end{array}\right]
$$

then Eq. (9) may be written

$$
m_{i, j}^{\prime}=-\left(S^{\prime} \omega_{i j}^{\prime}-C^{\prime} S^{\prime} \omega_{j i}^{\prime}\right)
$$

Let the rotation vector of a joint, if referred to the $(\mathrm{x}, \mathrm{y})$ coordinate sys-

Where possible, the subscripts $i$ and $j$, which identify the member but do not indicate components, will be dropped if no lack of clarity results from this omission. 

tem, be designated by $\omega$, and let similarly the moment vector $m$ refer to the same system, or

$$
m=\left[\begin{array}{l}
m_{x} \\
m_{y}
\end{array}\right] \quad \omega=\left[\begin{array}{c}
\omega_{x} \\
\omega_{y}
\end{array}\right]
$$

It follows then, from considerations of statics and geometry, respectively, that

$$
\begin{array}{ll}
m^{\prime}=R m & \omega^{\prime}=R \omega \\
m=R^{*} m^{\prime} & \omega=R^{*} \omega^{\prime}
\end{array}
$$

in which the rotation matrix $R$ and its transpose $R^{*}$ are defined by

$$
R=\left[\begin{array}{cc}
\cos \alpha & -\sin \alpha \\
\sin \alpha & \cos \alpha
\end{array}\right]
$$

When Eqs. (15) and (14) are substituted in Eq. (13), the "slope-deflection" equations in the $(x, y)$ coordinate system read

$$
m_{i j}=-\left(S \omega_{i}+C S \omega_{j}\right)
$$

In Eq. (17), $\mathrm{S}$ and $\mathrm{C}$ are the stiffness and carry-over matrices in the new coordinate system, that is,

$$
S=\left[\begin{array}{cc}
S_{x x} & S_{x y} \\
S_{y x} & S_{y y}
\end{array}\right] \quad C=\left[\begin{array}{cc}
C_{x x} & C_{x y} \\
C_{y x} & C_{y y}
\end{array}\right]
$$

which are related to the previous (primed) coordinate system by means of the transformation equations

$$
S=R * S \cdot R \quad C=R^{*} C^{\prime} R
$$

Hence, by Eqs. (12), (18), and (19), and after some trivial trigonometric transformations: 



$$
\begin{aligned}
& S_{x x}=1 / 2\left(S_{t}+S_{m}\right)+1 / 2\left(S_{t}-S_{m}\right) \cos 2 \alpha \\
& S_{y y}=1 / 2\left(S_{t}+S_{m}\right)-1 / 2\left(S_{t}-S_{m}\right) \cos 2 \alpha \\
& S_{x y}=S_{y x}=-1 / 2\left(S_{t}-S_{m}\right) \sin 2 \alpha
\end{aligned}
$$

and an equivalent set of equations for the components of $\mathrm{C}$. Since furthermore $\mathrm{R}$ is orthogonal, i.e., $R^{*}=I$ (the unit matrix), the matrix C'S' itself transforms similarly to Eq. (20). Finally, in developing Eq. (17), use was made of the fact that $\alpha_{j i}=\alpha_{i j}+\pi$ and that therefore $R_{i j}=-R_{j i}$; hence, in the new coordinate system, the $i, j$ subscripts continue to be interchangeable for the stiffness and carryover matrices.

In review, the relationships developed above insure that the end rotations $\beta_{i j}$ and $\theta_{i j}$ of all bars are compatible with a set of joint rotation vectors $\omega_{i}$. The establishment of the equations of equilibrium is now straightforward, since Eq. (17) expresses all the bar moment vectors with reference to the common (x,y) coordinate system. If $\mathrm{n}$ is the number of joints that are free to rotate, the following $n$ vector equations (or $2 n$ scalar equations) follow therefore immediately:

$$
\sum_{j} m_{i j}=0(i=1,2 \ldots n)
$$

where the summation extends over all the bars joined together at $i$.

The equations of equilibrium (21) now lead directly to the determinantal stability criterion. In fact, define the "total joint stiffness" $\mathrm{K}_{\text {ii }}$ by

$$
K_{i i}=\sum_{j} S_{i . j}
$$

and let further

$$
K_{i j}=C_{i j} S_{i j} \quad(i \neq j)
$$



Then substitution of Eq. (17) in Eqs. (21) results in the set of linear homogeneous equations

$$
\sum_{j} K_{i j} \omega_{j}=0(i=1,2 \ldots n)
$$

This system has a nontrivial solution if, and only if, the $2 \mathrm{n} x 2 \mathrm{n}$ determinant of the coefficients of the joint rotations vanishes. The structure is therefore in neutral equilibrium under the smallest load for which

$$
\left|K_{i j}\right|=0
$$

\section{A SERIES CRITERION OF STABILITY}

Eq. (24) represents a highly involved transcendental equation in terms of the external loads; to find its smallest root (that is, the smallest multiplier common to all loads) is therefore a task of forbidding numerical complexity. In this section, an alternate approach is presented, which constitutes a generalization of the "series criterion" of stability, which was first proposed by Lundquist [4] in connection with the stability of frameworks against buckling in their own plane.

Briefly, the suggested method proceeds as follows. Select a joint q (preferably the one most likely to buckle) and apply at $q$ a unit moment about the $x-$ axis. By means of a generalized moment distribution process which will be described in detail further below, this moment is distributed among the bars joined at $q$. This introduces unbalanced moments, generally about both the $x$ and $y$ axes, at the far ends of these bars. Holding now joint q fixed, these moments are all distributed in successive steps until all the other joints are balanced. If this process converges, 7 the only unbalanced joint will finally be the original joint 7 In case of divergence, the structure is known to be unstable; in fact, it is unstable even with joint q fixed. 

$q$, toward which the residual moments $r_{x x}$ and $r_{y x}$ about the $x$ - and $y$-axis, respectively, have been "washed back." If the same procedure is now repeated for an original applied unit moment about the y-axis at $q$, the wash-back moments will be, say, $r_{x y}$ and $r_{y y}$. Note that, in general, $r_{x y} \neq r_{y x}$.

If now the "residual matrix" $r$ is defined by

$$
r=\left[\begin{array}{cc}
r_{\mathrm{xx}} & r_{\mathrm{xy}} \\
r_{\mathrm{yx}} & r_{\mathrm{yy}}
\end{array}\right]
$$

then the structure is in general ${ }^{8}$ stable if, and only if, the matrix I - $r$ has a positive trace and determinant; hence

$$
\begin{array}{r}
r_{x x}+r_{y y}<2 \\
\left(1-r_{x x}\right)\left(1-r_{y y}\right)-r_{x y} r_{y x}>0
\end{array}
$$

constitutes the desired series criterion of stability.

Suppose now that, for a given load parameter, the procedure described above yields a residual matrix which satisfies conditions (26); the structure is then known to be stable for this sytem of loads, and the process is repeated for a larger load multiplier, until one or the other of the conditions is violated. The exact value of the load parameter associated with neutral equilibrium can normally be estimated with a fair degree of accuracy through interpolation.

The joint balancing procedure itself represents an extension and generalization of the familiar moment distribution method; the employment of matrix notation makes it possible to describe it in almost identical terms. In fact, let the joint $i$ undergo a rotation (vector) $\omega_{i}$, while all other joints remain fixed. It then follows, from Eq. (17), that each bar $(i, j)$ exerts a moment $-S_{i j} \omega_{i}$ on

8 A possible, though unlikely, exception occurs when the lowest buckling mode does not involve any joint rotation of $q$ about either axis. In that case, Eqs. (26) constitute a necessary, but not sufficient condition of stability. 

this joint, while at the far end of the bars, say at $(j)$, an unbalanced moment $-(\mathrm{CS})_{i j} \omega_{i}$ is introduced. It may be worth repeating, at this time, that all the vectors and matrices are expressed in relation to their common ( $x, y)$ coordinate system.

If now the rotation $\omega_{i}$ is so chosen as to balance the joint, it follows that all the new bar moments at $i$ must equal the existing unbalanced moment $m_{i}^{u}$. If, further, the definition of the total joint stiffness $K_{i i}$ is recalled as given by Eq. (22a), the required joint rotation must be equal to $\left(K_{i i}\right)^{-1} m_{i}^{u}$. Hence, whenever an unbalanced moment $\mathrm{m}_{i}^{u}$ is removed at the joint $i$, while all the other joints remain temporarily fixed, the share taken by each bar at $i$ is given by

$$
m_{i j}=-S_{i j}\left(K_{i i}\right)^{-1} m_{i}^{u}
$$

while the new unbalanced moment introduced at the far end $j$ of the bar is governed by

$$
m_{j}^{u}=C_{i j} m_{i j}
$$

The matrix $\mathrm{S}_{i j}\left(\mathrm{~K}_{i j}\right)^{-1}$ in $\mathrm{Eq}$. (27) may be interpreted as a "generalized distribution factor," while $\mathrm{C}_{i j}$ in $\mathrm{Eq} .(28)$, as mentioned before, represents a "generalized carry-over factor." In the problem under discussion, in which stability rather than stresses are being investigated, some saving in labor can be effected by combining steps (27) and (28) - that is, by transferring unbalanced moments directly from one joint to the other joints. This is achieved by letting

$$
m_{j}^{u}=-T_{i j} m_{i}^{u}
$$

in which the "transfer matrix" $T_{i j}$ is defined by

$$
T_{i j}=C_{i j} S_{i j}\left(K_{i j}\right)^{-1}
$$

Note that this matrix is generally not symmetrical, nor is, in general, $\mathrm{T}_{i j}$ equal 

to $\mathrm{T}_{j i}$

The numerical computation of the transfer matrices (two for each member of the framework) and the ensuing moment distribution procedure are reasonably simple and can be carried out on the slide rule. After all the matrices $S$ and CS have been determined by means of Eqs. (20), the total joint stiffnesses $\mathrm{K}$ are calculated by adding the corresponding components of the stiffness matrices for any one joint. Letting $\mathrm{K}_{\mathrm{Xx}}, \mathrm{K}_{\mathrm{Xy}}=\mathrm{K}_{\mathrm{yx}}$, and $\mathrm{K}_{\mathrm{yy}}$ be the components of $\mathrm{K}$ for a given panel point, the inverse (flexibility) matrix $\mathrm{K}^{-1}$ is given by

$$
K^{-1}=1 / k\left[\begin{array}{cc}
K_{y y} & -K_{x y} \\
-K_{x y} & K_{x x}
\end{array}\right] \quad k=K_{x x} K_{y y}-K_{x y}^{2}
$$

It is presumed here that all total joint stiffness matrices are positive definite, since otherwise the structure is known to be unstable, necessitating a (probably substantial) reduction in the load factor. Hence, $k \neq 0$ and $\mathrm{K}^{-1}$ exists.

Let it now be assumed that the two moment distribution procedures have been carried out and the residual matrix $r$ has been established as described at the beginning of this section. It is evident that if, instead of two unit moments, an arbitrary moment $\mathrm{m}$ of components $\mathrm{m}_{\mathrm{x}}$ and $\mathrm{m}_{\mathrm{y}}$ were applied at joint $\mathrm{q}$, the residual moment vector would be $\mathrm{rm}$. Since further $\mathrm{m}=\mathrm{K}$, it follows that $(I-r) K$ is the actual moment introduced at $q$. In other words, ( I-r)K represents the equivalent stiffness of the joint $q$ if all other joints are free to rotate. The positive definiteness of this matrix is therefore a necessary condition for the stability of the structure. The sufficiency condition, which is subject to the exception noted before, 8 can be proved by extending the argument employed in a similar problem in [5].

From this criterion of positive definiteness, conditions (26) can be derived directly. Use is made here of the symmetry of $(I-r) K$, which is a consenquence of 

Maxwell's Theorem of Reciprocity and which, incidentally, also provides some means of checking the numerical work. It follows that the stability of the structure depends on the positiveness of the trace and determinant of $(I-r) K$. As for the latter, since $\mathrm{K}$ itself has a positive determinant, $|I-r|$ must also be positive. In regard to the trace, let the coordinate system be rotated so that $K$ assumes a diagonal form; this is always possible on account of its symmetry. By examining the form of $(I-r) K$ in this coordinate system, and by considering further that the diagonal terms of $\mathrm{K}$ are both positive, it can readily be verified that ( $I-r) K$ has a positive trace if, and only if, the trace of (I-r) itself is positive in this, and hence in the original, coordinate system. This completes the proof of the inequalities (26).

\section{NUMERICAL EXAMPLE}

The method outlined above is now applied to the truss shown in Fig. 3. All joints are connected rigidly and are prevented from lateral sway by means of cross bracing (not shown), which, however, does not contribute to their rotational stiffness. All gusset plates are assumed to be $1 / 2$ " thick.

The purpose of the study is to determine the factor of safety against buckling-that is, the factor by which the given load must be multiplied to render the equilibrium of the structure neutral. In particular, it will be shown that although the compression members have been so selected as to make their $\mathrm{L} / \mathrm{r}$ ratios about equal, the danger of buckling out of the plane of the truss is considerably greater than that within the plane,at least so far as the structure under consideration is concerned.

As for the stability within the plane of the framework, the investigation proceeds along the familiar lines as outlined originally in [4]. The applied load $\mathrm{W}=50,000 \mathrm{lb}$ was multiplied by several assumed values of the safety factor and, for 

each value, the stiffness and carry-over factors were computed with the aid of [3]. A unit moment in the plane of the truss was then applied at joint B, distributed among the three members joined at B, and, with B fixed, the remaining joints were balanced. If the effect of yielding is disregarded, it was found that the residual moment at joint $B$ is 0.962 for a safety factor of 4.50 , and 1.066 for 4.60. Hence, the load corresponding to plane instability lies between 225,000 and $230,0001 b$.

The investigation of the lateral stability proceeded along an analogous path, as described in the previous section. For an assumed load multiplier, the axial bar forces were computed as well as the equivalent forces as given in Eqs. (3) and (6). The bending stiffness and carry-over factors $S_{m}$ and $C_{m}$ were next determined, as in the case of plane buckling. For the example of the double-angle section as used here, a measure of simplification is introduced by the fact that the torsional stiffness $S_{t}$ is negligible in comparison with $S_{m}$. The determination of the S and CS matrices in the (x,y) coordinate system, as given by Eqs. (20), is simplified accordingly.

Table I shows a representative portion of the constants involved for an assumed safety factor of 2.6. The material constants $E$ and $G$ were assumed to be equal to $29 \times 10^{6} \mathrm{psi}$ and $11.6 \times 10^{6} \mathrm{psi}$, respectively. Since only relative stiffnesses are of concern in connection with this study, a number of common factors were disregarded in the establishment of $\mathrm{S}_{\mathrm{m}}$. However, it is important to retain the correct proportionality in computing $S_{t}$ (not needed here) from Eqs. (10), particularly since the tables in [3] give values of $\mathrm{S}_{\mathrm{m}}$ which are only one-fourth of those corresponding to Eqs. (10).

The total joint stiffness is now determined by adding the corresponding components of the $S$ matrices of all the bars connected at a particular joint. For example, the stiffness matrix $K_{A}$ of panel point $A$ is obtained as the sum of $S_{A B}$ and $\mathrm{S}_{\mathrm{AD}}$; this leads to 



$$
K_{A}=\left[\begin{array}{ll}
0.45 & 0.45 \\
0.45 & 1.29
\end{array}\right]
$$

which is positive definite and has an inverse [see Eq. (30)]

$$
\mathrm{K}_{\mathrm{A}}^{-1}=\left[\begin{array}{rr}
3.40 & -1.29 \\
-1.29 & 1.29
\end{array}\right]
$$

The stiffness matrices of all the other joints, and their inverse, are then computed in the same fashion.

TABLE I

\begin{tabular}{|c|c|c|c|c|c|c|c|c|c|c|c|c|}
\hline \multirow{2}{*}{ Bar } & \multirow{2}{*}{ Force } & \multirow{2}{*}{$\mathrm{N}$} & \multirow{2}{*}{$\varnothing$} & \multirow{2}{*}{$\mathrm{C}_{\mathrm{m}}$} & \multirow{2}{*}{$\mathrm{S}_{\mathrm{m}}$} & \multirow{2}{*}{$\alpha$} & \multicolumn{3}{|c|}{$\mathrm{S}$} & \multicolumn{3}{|c|}{ CS } \\
\hline & & & & & & & $\mathrm{xx}$ & $x y$ & yy & $x x$ & $x y$ & yy \\
\hline$A B$ & 91.9 & 105.2 & $3.62 \mathrm{C}$ & 1.48 & 0.90 & 45 & .45 & .45 & .45 & .67 & .67 & .67 \\
\hline $\mathrm{CB}$ & 91.9 & 105.2 & $3.62 \mathrm{C}$ & 1.48 & 0.90 & 135 & .45 & -.45 & .45 & .67 & -.67 & .67 \\
\hline $\mathrm{BD}$ & -130 & -118 & $2.70 \mathrm{~T}$ & 0.37 & 3.40 & -90 & 3.40 & 0 & 0 & 1.26 & 0 & 0 \\
\hline $\mathrm{AD}$ & -65 & -63.4 & $4.16 \mathrm{~T}$ & 0.26 & 0.84 & 0 & 0 & 0 & .84 & 0 & 0 & .22 \\
\hline$C D$ & -65 & -63.4 & 4.1611 & 0.26 & 0.84 & 180 & 0 & 0 & .84 & 0 & 0 & .22 \\
\hline
\end{tabular}

STIFFNESS AND CARRY-OVER CONSTANTS FOR A SAFETY FACTOR OF 2.6

The final step consists in the establishment of the transfer matrjces defined by Eqs. (29). This is achieved by multiplying the CS matrix of a member, as given in Table $I$, by the inverse stiffness matrix of the joint from which the unbalanced moment is to be transferred. For example, ${ }^{\mathrm{T}} \mathrm{AB}$ represents the effect of the balancing of joint $A$ on the unbalanced moment at $B$; it is given by

$$
\mathrm{T}_{\mathrm{AB}}=(\mathrm{CS})_{\mathrm{AB}} \mathrm{K}_{\mathrm{A}}^{-1}=\left[\begin{array}{ll}
.67 & .67 \\
.67 & .67
\end{array}\right]\left[\begin{array}{rr}
3.40 & -1.29 \\
-1.29 & 1.29
\end{array}\right]=\left[\begin{array}{ll}
1.42 & 0 \\
1.42 & 0
\end{array}\right]
$$



It should be emphasizeã once again that two such transfer matrices must be computed for each bar since, in general, $\mathrm{T}_{\mathrm{AB}} \neq \mathrm{T}_{\mathrm{BA}}$.

Fig. 4 shows all the transfer coefficients for a safety factor of 2.6; it also demonstrates the moment distribution procedure, which in this case turns out to be exceptionally simple owing to the symmetry of the structure. A brief description at this point, it is felt, may elucidate the simple, but perhaps somewhat unfamiliar steps. The introduction and balancing of a unit moment about the $\mathrm{x}$-axis at point $\mathrm{B}$ produces unbalanced moments about both axes at joints $A, D$, and $C$, as shown in the first line. When the unbalanced moment $m_{X}=-0.15$ at $\mathrm{A}$ is removed, the moments about both axes, which are transferred back to $\mathrm{B}$, are each equal to $-(1.42)(-0.15)=+0.21$; at the same time, a moment vector is transferred to joint $\mathrm{D}$ whose $\mathrm{x}$-component is zero and whose $\mathrm{y}$-component is given $-(-0.28)(-0.15)=-0.04$. Similarly, the balancing of $m_{y}=-0.15$ at $\mathrm{A}$ produces $(0,0)$ at $B$ and $(0,+0.04)$ at $D$.

The process is repeated for joint $\mathrm{C}$, with analogous results. The unbalanced moments at $D$ now are -0.29 and 0 , respectively, about the $x$-and $y$-axes. The removal of the former introduces an unbalancing effect only at joint B, to which an additional moment vector is transferred, whose $x$-component equals $-(0.37)(-0.29)=$ 0.11 and whose $\mathrm{y}$-component vanishes.

With all joints other than $B$ balanced, it is seen that the latter is now subjected to a residual unbalanced moment whose components are 0.53 and 0 , respectively. If the same process is carried out after an initial unit moment about the $y$-axis is balanced at $B$, it can be demonstrated that the residual moment vector is given by $(0,2.10)$. Hence, the residual matrix, as defined by Eq. (25), is

$$
r=\left[\begin{array}{ll}
0.53 & 0 \\
0 & 2.10
\end{array}\right]
$$

which is seen to violate the stability criterion (26). In other words, the struc- 

ture is laterally unstable under a load of $130,000 \mathrm{lb}$.

The same procedure, if carried out for a safety factor of 1.8 (corresponding to a load of $90,000 \mathrm{lb})$, leads to

$$
r=\left[\begin{array}{ll}
0.35 & 0 \\
0 & 0.80
\end{array}\right]
$$

which satisfies both criteria (26). The actual factor of safety of the structure is therefore known to lie between 1.8 and 2.6. While its exact value is not important in connection with the general discussion in this paper, it does seem noteworthy that it is very significantly smaller than its counterpart against plane buckling.

\section{CONCLUSION}

As pointed out already in the Introduction, the present theory is based on the assumption that, prior to lateral buckling, all members are subjected to axial. forces through their centroids. What effect, if any, the presence of so-called secondary moments in the plane of the structure has on the condition of lateral stability is difficult to assess without considering a specific example. It is known, however [6], that structures whose bending moments are statically indeterminate do not ordinarily collapse under loads which are associated with incipient lateral instability. The type of framework under consideration represents such an example; hence, it is reasonable to expect the actual collapse load to exceed the theoretical buckling load.

In any event, the factor of safety which is computed on the basis of the simplifying assumption of this paper must represent a lower bound to the factor of safety based on the collapse load. Indeed, as pointed out in [6], any safety factor based on statically admissible bending moments represents such a lower 

bound; obviously, a set of identically vanishing bending moments is statically consistent in the absence of loads other than those applied at the joints.

Whether the startlingly large discrepancy between the safety factors against buckling in and out of the plane of the structure can be extended to trusses of different geometry, or of greater torsional resistance, or composed of members heavy enough to correspond to plastic buckling, can of course not be answered summarily at this point. On the other hand, the results of this study seem to indicate that the current design practice of equalizing the slenderness ratios of compression members in both directions may not necessarily lead to an efficient functioning of the structure. Perhaps it may not be unreasonable to suggest that the pertinent features of the present code be subjected to further scrutiny in this respect.

\section{BIBLIOGRAPHY}

1. "Steel Construction," American Institute of Steel Construction, 5tr Ed., 1950.

2. "Buckling Strength of Metal Structures," by F. Bleich, McGraw-Hill Book Co., Inc., 1952.

3. "Tables of Stiffness and Carry-Over Factors for Structural Members under Axial Load," by E. E. Lundquist and W. D. Kroll, NACA Tech. Note 652, 1938.

4. "Stability of Structural Members under Axial Load," by E. E. Lundquist, NACA Tech. Note 617, 1937.

5. "On the Fundamental Frequencies of Vibration of Rigid Frames,"by E. F. Masur, Proceedings of the First Midwestern Conference on Solid Mechanics, p. 89-94, Urbana, 1953.

6. "The Collapse Strength of Redundant Structures after Lateral Buckling," by E. F. Masur and K. P. Milbradt, submitted for publication to Journal of Applied Mechanics, ASME. 



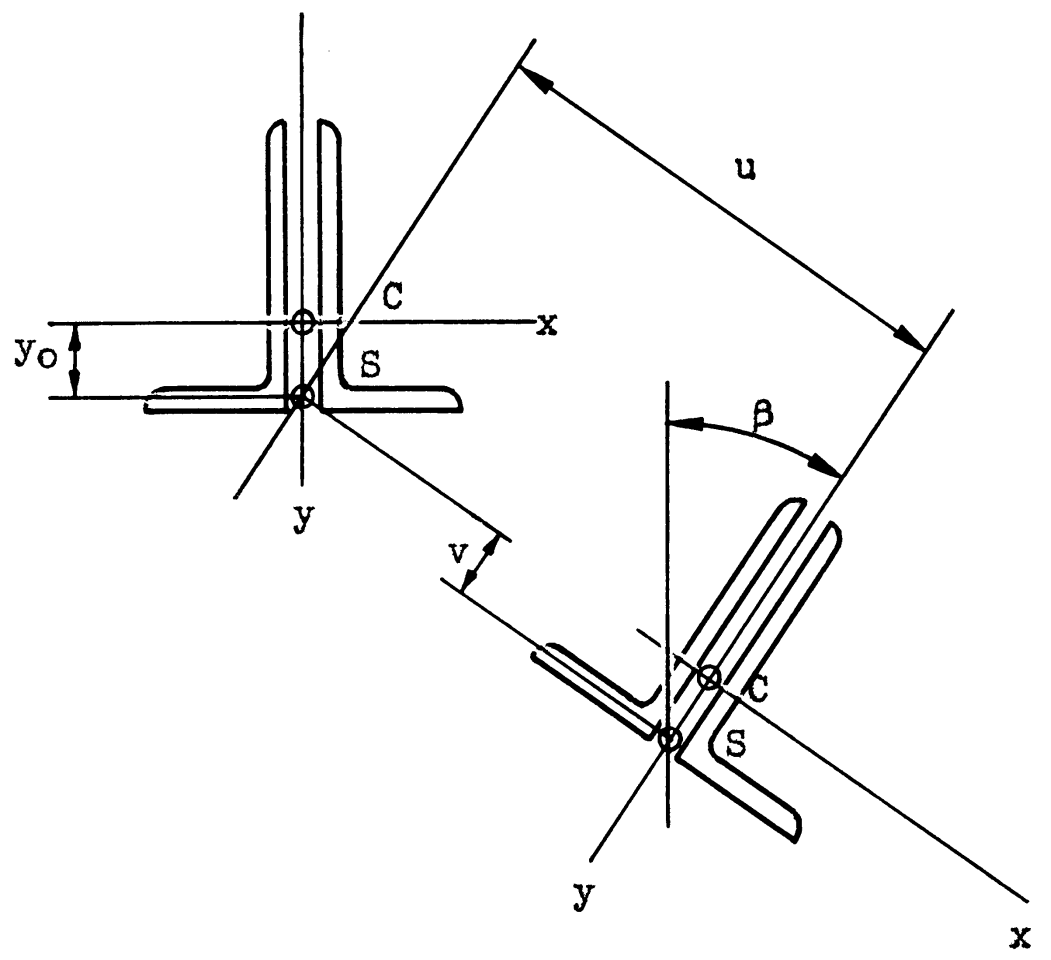

Fig. 1

Typical buckled cross section.

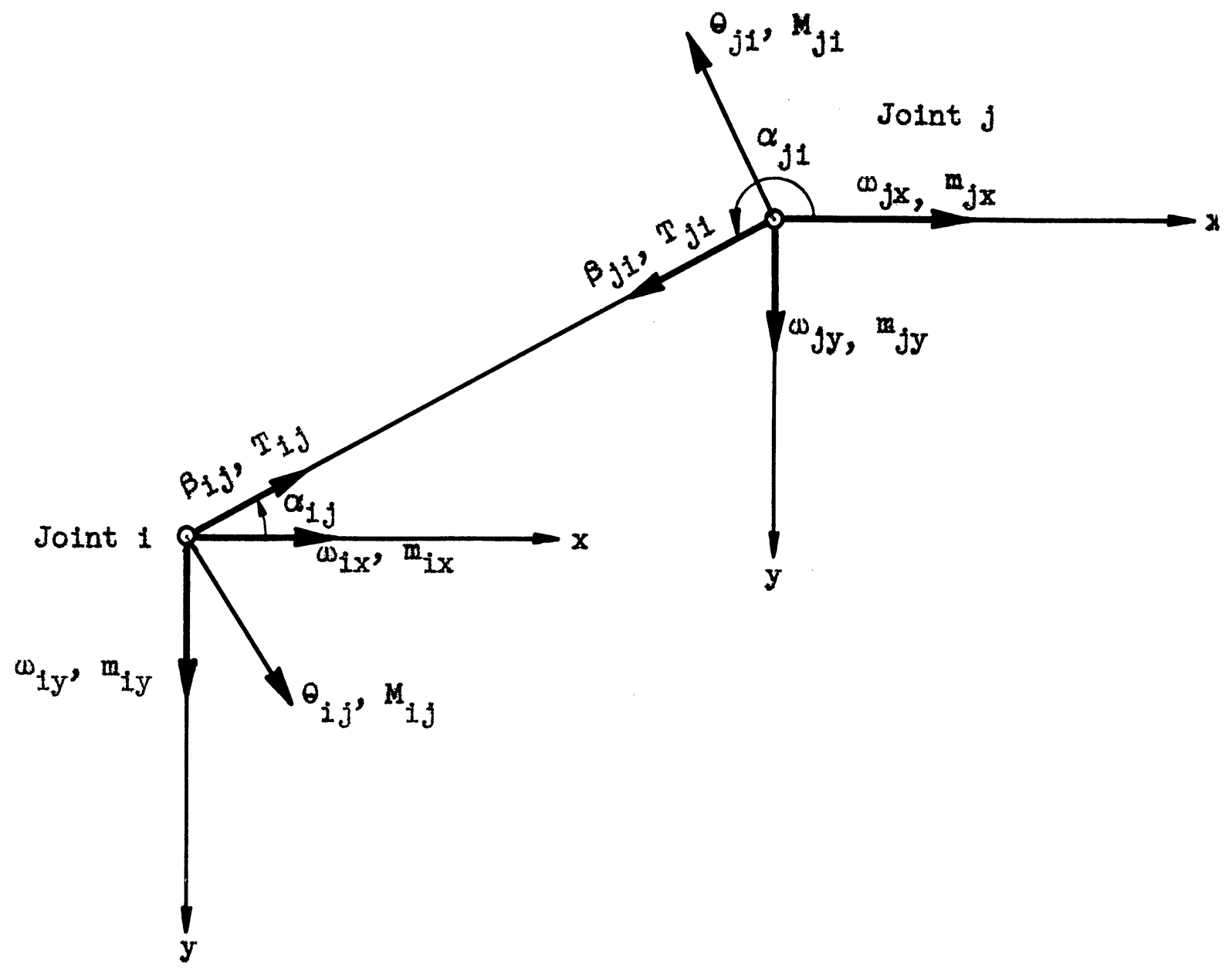

Fig. 2

Positive rotation and moment vectors in representative bar. 



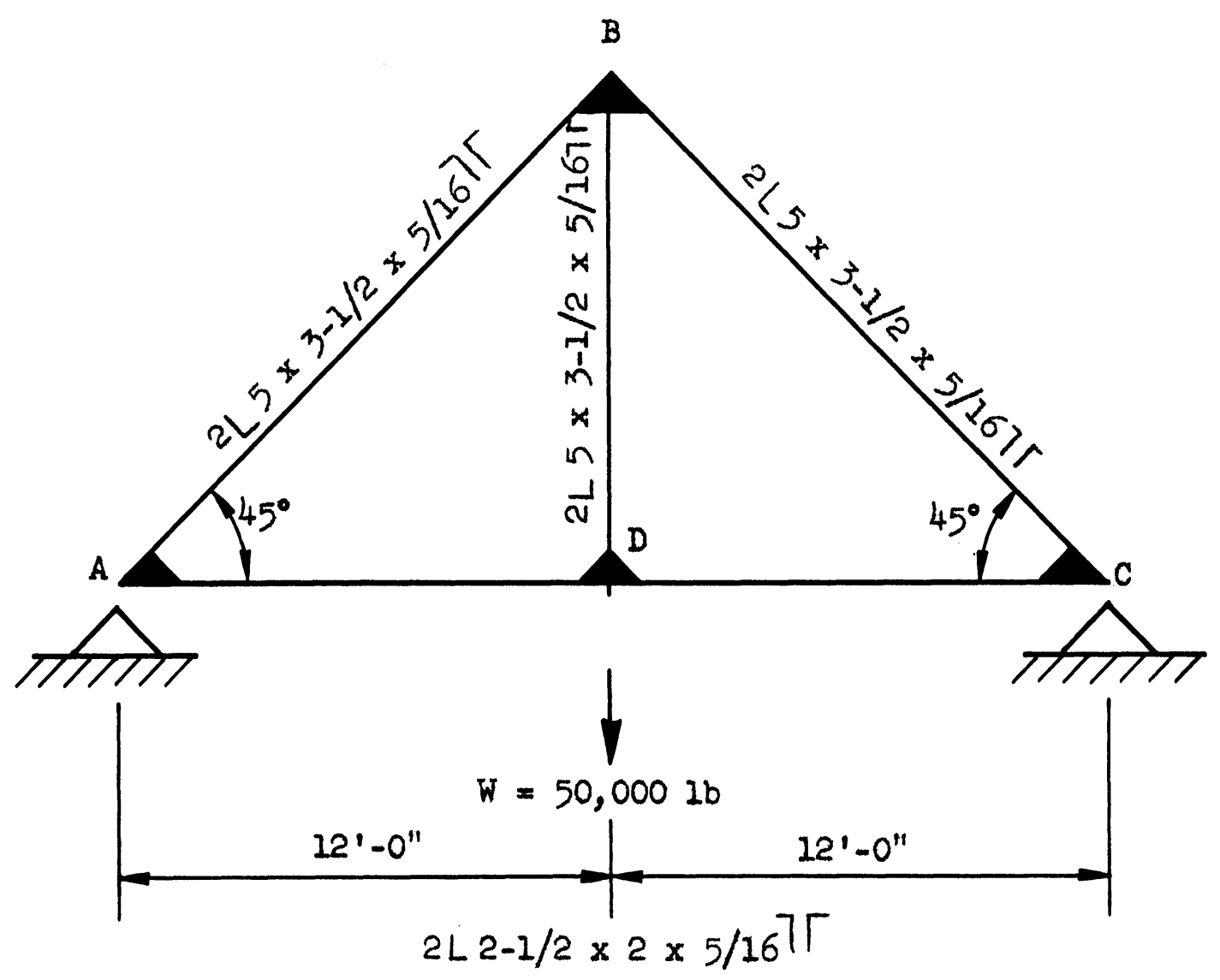

Fig. 3

Example of framework.

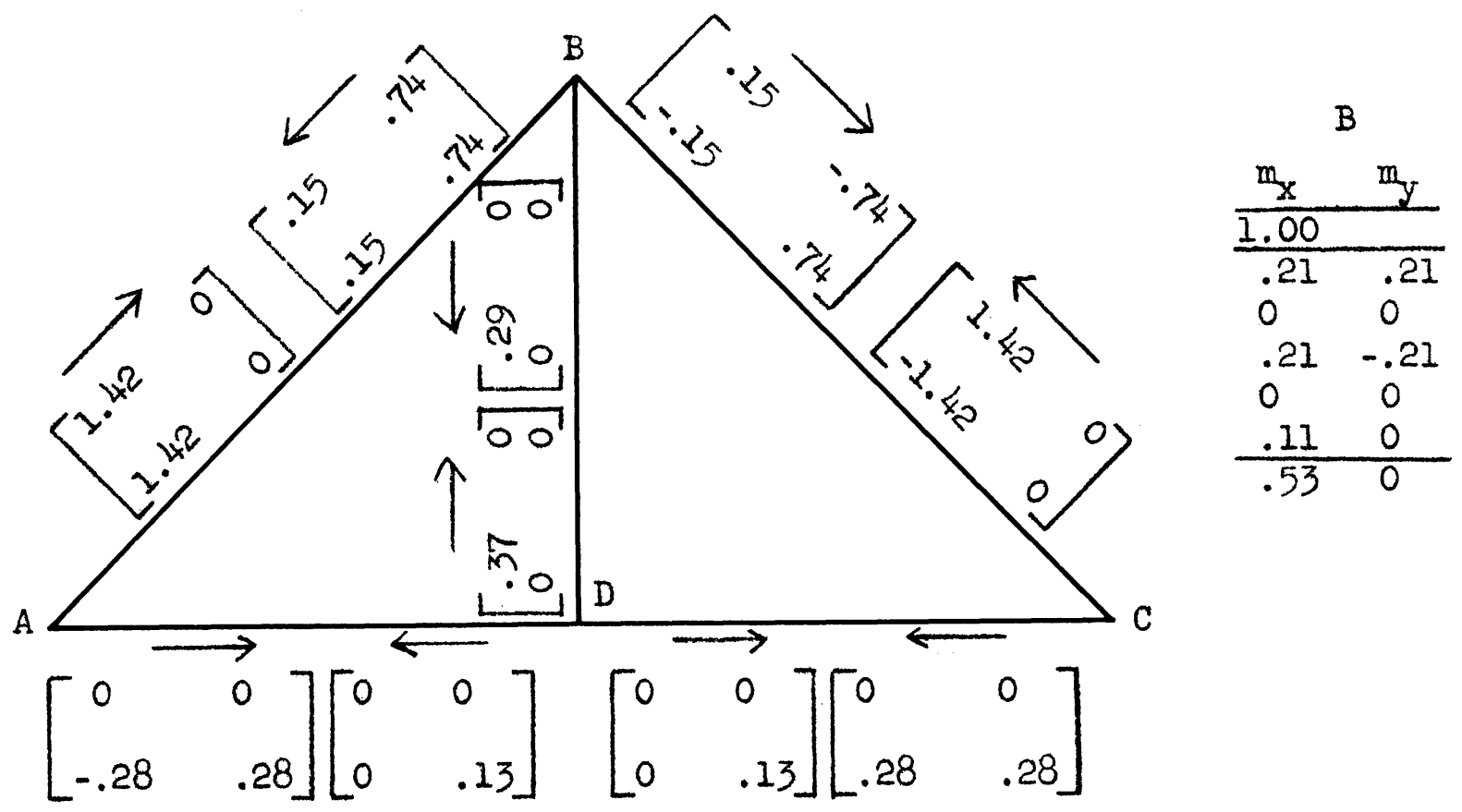

A

D

\begin{tabular}{rr}
$\mathrm{m}_{\mathrm{x}}$ & $\mathrm{m}_{\mathrm{y}}$ \\
\hline. .15 & -.15 \\
\hline
\end{tabular}

\begin{tabular}{cc}
$\mathrm{m}_{\mathrm{x}}$ & $\mathrm{m}_{\mathrm{y}}$ \\
\hline-.29 & 0 \\
0 & -.04 \\
0 & +.04 \\
0 & +.04 \\
0 & -.04 \\
\hline
\end{tabular}

\begin{tabular}{rr}
$m_{x}$ & $m_{y}$ \\
\hline .15 & .15 \\
\hline
\end{tabular}

Fig. 4

Moment distribution. 



\section{DISTRIBUTION LIST}

Contract No. DA-20-018-ORD-14085

Commanding Officer

Office of Ordnance Research

Box CM, Duke Station

Durham, North Carolina

Chief of Ordnance

Department of the Army

Washington 25, D. C.

Attn: ORDTB-PS

Deputy Chief of Staff for Logistics

Department of the Army

Washington 25, D. C.

Attn: Research Branch, $R$ and $D$ Division

Commanding General

Aberdeen Proving Ground

Maryland

Attn: Tech. Info. Division

Commanding Officer

Detroit Arsenal

Center Line, Michigan

Commanding General

Frankford Arsenal

Bridesburg Station

Philadelphia 37, Penn.

Attn: ORDBA-LC

Commanding General

Redstone Arsenal

Huntsville, Alabama

Attn: ORDDW-MR

Commanding General

White Sands Proving Ground

Las Cruces, New Mexico

Attn: ORDBS-TS-TIB

Commanding General

Ordnance Weapons Command

Rock Island, Illinois

Attn: Research Branch

Chief

Detroit Ordnance District

574 East Woodbridge

Detroit 31, Michigan

Attn: ORDEF-IM
Chief of Ordnance

Department of the Army

Washington 25, D. C .

Attn: ORDGU-SE

For Transmittal to:

Canadian Joint Staff

2001 Connecticut Ave., N.W.

Washington 25, D. C.

Office of Naval Research

Washington 25, D. C .

Attn: Code 438

Commander

U. S. Naval Proving Ground

Dahlgren, Virginia

U. S. Naval Ordnance Laboratory White Oak, Silver Spring 19, Ma.

Attn: Library Division

Chief, Bureau of Ordnance (AD3) I

Department of the Navy

Washington 25, D. C.

Director, Air University Library 1

Maxwell Air Force Base, Ala.

Commanding General

Air Research and Development Command, P. O. Box 1395

Baltimore 3, Maryland

Attn: RDTOIL (Tech. Library)

Commanding Officer

Engineering Res. and Dev. Labs.

Fort Belvoir, Virginia

1

Jet Propulsion Laboratory

California Institute of Tech. 4800 Oak Grove Drive

Pasadena 3, California

2

Attn: A. J. Stosick

Corona Laboratories

National Bureau of Standards

Corona, California

2

Technical Information Service

P.O. Box 62

Oak Ridge, Tennessee

Attn: Reference Branch 

DISTRIBUTION LIST (concluded)

Contract No. DA-20-018-ORD-14085

The Director

1

Snow, Ice and Permafrost

Research Establishment

Corps of Engineers

1215 Washington Avenue

Wilmette, Illinois

Armed Services Technical

Information Agency

Document Service Center

Knott Building

4 th and Main Streets

Dayton 2, Ohio

U. S. Atomic Energy Commission

1

Document Library

19th and Constitution Avenue, N.W.

Washington 25, D. C.

Director, Applied Physics Laboratory

1

Johns Hopkins University

8621 Georgia Avenue

Silver Spring 19, Maryland

Attn: Dr. R. C. Herman

Director

National Bureau of Standards

Washington 2.5, D. C. 




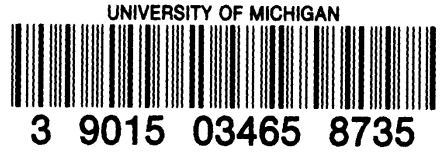

